\title{
Development of the Sasquatch Drop Test Footprint Tool
}

\author{
Kristin J. Bledsoe ${ }^{1}$ \\ Jacobs Engineering, Houston, TX, 77058
}

\begin{abstract}
The Crew Exploration Vehicle Parachute Assembly System (CPAS) is the parachute system for NASA's Orion spacecraft. CPAS is currently in the design and testing phase of development. The test program consists of numerous drop tests, wherein a test article rigged with parachutes is extracted or released from an aircraft. During such tests, range safety is paramount, as is the recoverability of the parachutes and test article. It is crucial to establish an aircraft release point that will ensure that the article and all items released from it will land in safe locations. Early in the CPAS project, a legacy tool (previously used on the X-38 project) was used to determine a safe release point and to predict the landing locations (the footprint) of the payload and all released objects. Due to increasing test complexity and the need for a more flexible tool, a new footprint predictor tool, called Sasquatch, was created in MATLAB. This tool takes in a simulated trajectory for the test article, information about all released objects, and atmospheric wind data (simulated or actual) to calculate the trajectories of the released objects. Dispersions are applied to the landing locations of those objects, taking into account the variability of winds, aircraft release point, and object descent rate. A safe aircraft release point is determined based on the landing locations of the payload and released objects. The release point, landing locations, and dispersions are plotted on a simple map of the drop zone for easy reference. To date, Sasquatch has been used for thirteen drop tests. Comparing the predictions with actual test results has allowed for significant improvements in the tool's predictive capabilities, especially the incorporation of a well-correlated horizontal throw model. Intended future improvements to the tool include tighter dispersions on the landing locations, Monte Carlo capability, direct input from trajectory simulations, and a graphical user interface.
\end{abstract}

\section{Nomenclature}

$\begin{array}{ll}\text { CDT } & =\text { Cluster Development Test (series) } \\ \text { CEV } & =\text { Crew Exploration Vehicle } \\ \text { CPAS } & =\text { Crew Exploration Vehicle Parachute Assembly System } \\ \text { ESCG } & =\text { Engineering Services Contract Group } \\ \text { KTM } & =\text { Kineto Tracking Mount (camera) } \\ \text { MDT } & =\text { Main Development Test (series) } \\ \text { MSL } & =\text { Mean Sea Level }\end{array}$

\section{Introduction}

$\mathrm{T}$

The Crew Exploration Vehicle Parachute Assembly System (CPAS) is the parachute system for NASA's Orion spacecraft. CPAS is currently in the design and testing phase of development. ${ }^{1-3}$ The test program consists of numerous drop tests, wherein a test article rigged with parachutes is extracted or released from an aircraft. A sequence of parachutes is deployed throughout the trajectory. At the end of each phase of flight, parachutes are cut away from the payload and descend separately to the ground.

During such tests, range safety is of high importance, as is the recoverability of the parachutes and test article. It is crucial to establish an aircraft release point that will ensure that the article and all items released from it will land in areas that will not endanger people on the ground or cause damage to the test article. Early in the CPAS project, a legacy tool from the X-38 project was used to determine a safe release point and to predict the landing locations (the footprint) of the payload and all released objects. The code for that tool was not available for modification. Due to increasing test complexity and the need for more control over the code, a new footprint predictor tool, called

${ }^{1}$ Aerospace Engineer, CPAS Analysis Group, 2224 Bay Area Blvd, Houston, TX 77058, Non-member.

1

American Institute of Aeronautics and Astronautics 
Sasquatch, was created in MATLAB. Sasquatch has been used successfully for thirteen drop tests, providing predictions that correlate well with actual flight test results.

\section{Footprint Concerns}

There are two primary concerns with regards to test footprints: range safety and the recoverability of the test articles.

To date, all CPAS tests have been executed on designated drop test ranges. The Robby/LaPosa drop zone at the Yuma Proving Ground (YPG) is the range most commonly used, as it is large enough to contain all released items from drops as high as $25,000 \mathrm{ft}$ and has a cleared area that allows for safe recovery of the payloads. One of the primary concerns when testing on a designated range is the need to keep all items within the range boundaries. The LaPosa drop zone is bounded by a public highway on the east, other test areas on the south and north, and mountains on the west. The eastern boundary is the most significant; impinging on the highway endangers the safety of anyone traveling on the road. The winds on the drop zone are towards the east during most times of the year, rendering the concern even stronger. Other hazards near the drop zone include power lines to the south, camera positions to the west, and a staging area for drop zone personnel on the drop zone itself. In order to perform a drop test, there must be a reasonable expectation that the payload and released objects will not encounter any of these hazards or safety zones.

There is also a concern about the recoverability of test articles. Parachutes can be damaged by landing on or being dragged through vegetation such as bushes and cacti. A large area on the LaPosa drop zone has been plowed to clear it of foliage that might damage the test articles. The remaining obstacles that cannot be removed have recently been surveyed so that CPAS knows and can limit the possibilities of landing in an area that may damage the test hardware.

Day of test wind and atmosphere information is key to footprint calculations. The wind direction and speed

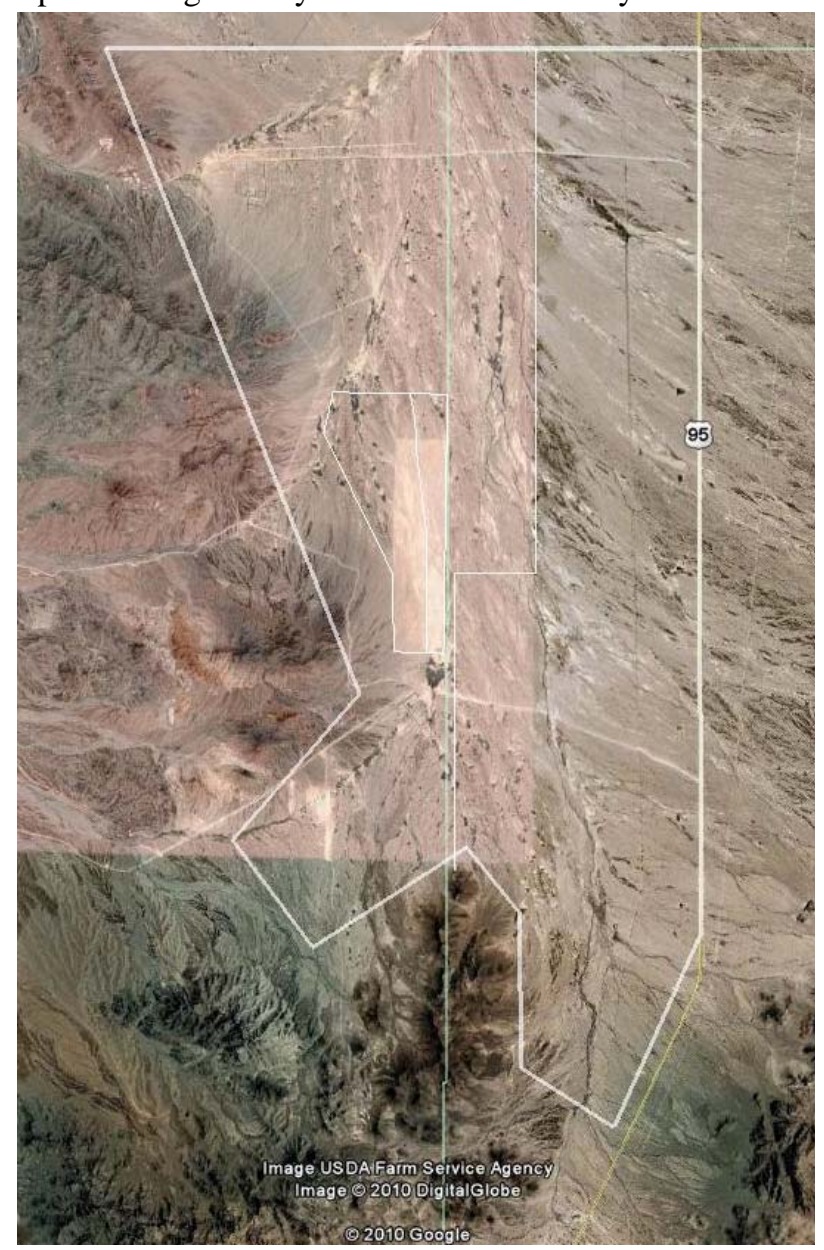

Figure 1. YPG Robby/LaPosa Drop Zone dictates the horizontal motion of the objects, while the air density affects the descent rates. Atmospheric data for CPAS tests are typically measured by Rawinsonde balloons. ${ }^{4}$ Balloons are sent up hourly on the morning of a test,
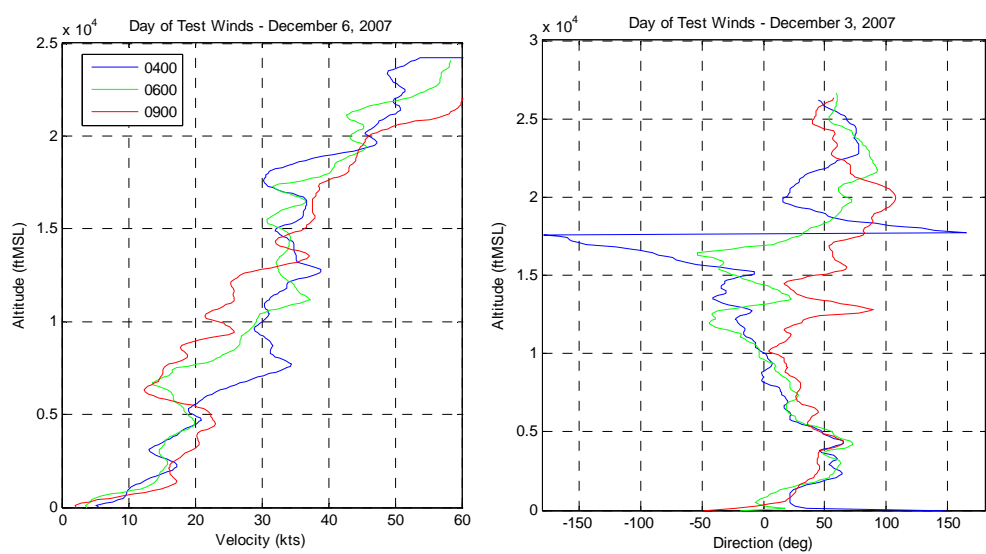
starting several hours before the intended drop time. Footprint runs are made early to determine feasibility of testing on that day. However, a single, early run is insufficient to ensure accurate predictions, as winds can shift significantly in direction and magnitude over the course of a few hours (Fig. 2). For the most accurate footprint predictions, wind data must be as current as possible. For CPAS, the final preflight balloon data is typically received no more than two hours before the test.

Figure 2. Wind Shift on Sample Test Days 


\section{Functionality}

\section{A. Structure}

Sasquatch consists of two main functions: driver.m and fp.m (Fig. 3). The driver takes the user inputs, reads in the appropriate files, calculates the release point, collects the calculated landing locations for all objects, and outputs the information to the screen. The calculation of the landing locations is done within fp.m.

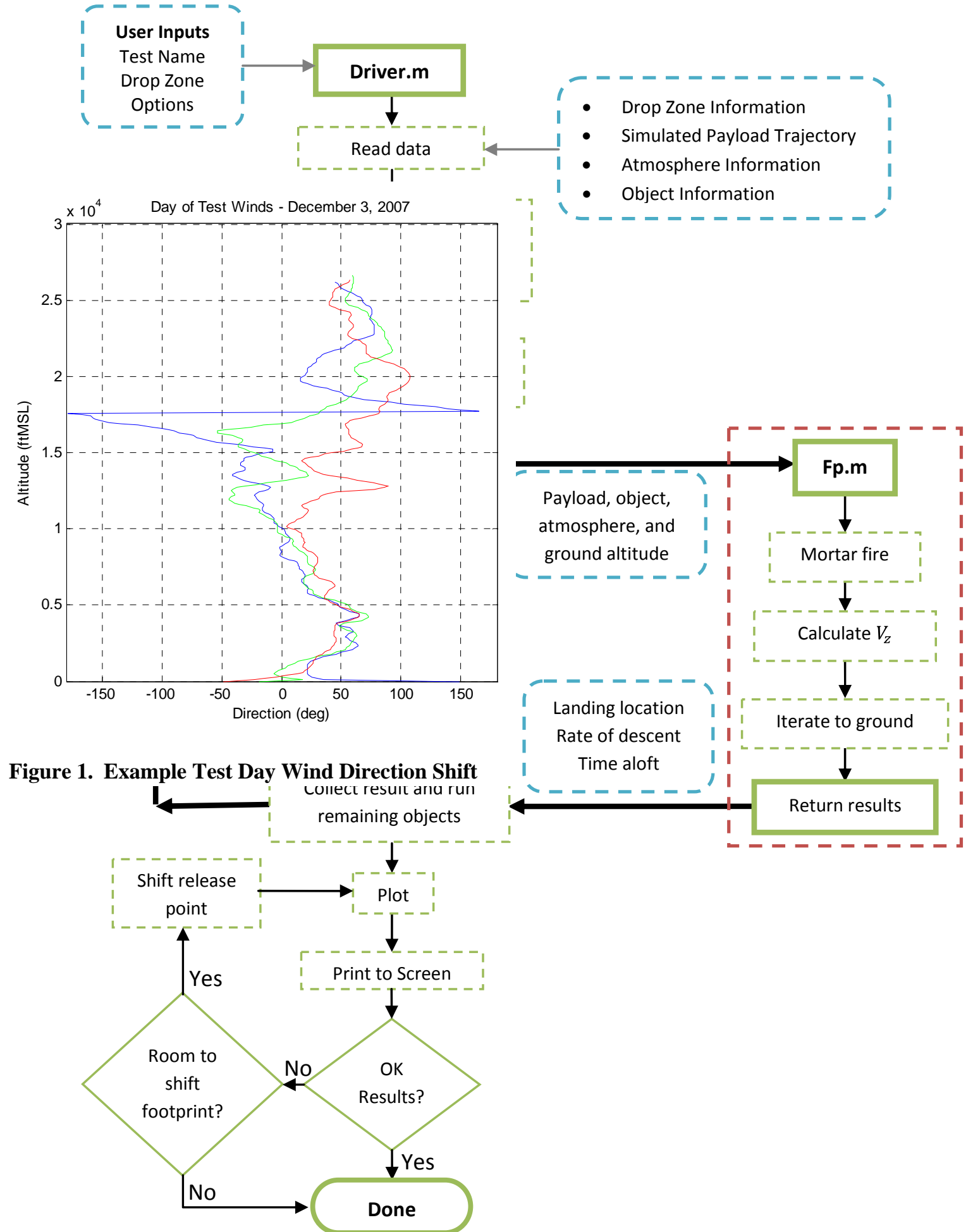

Figure 3. Sasquatch Structure

American Institute of Aeronautics and Astronautics 


\section{B. Inputs}

To run Sasquatch, the user calls driver.m, specifying the test name and the drop zone that will be used for the test. Additional options can also be included, such as the option to run only the footprint for a single object, options to include auxiliary objects (such as delivery systems and wind measurement devices), and plotting options.

Once Sasquatch initializes, it reads in four input files: a simulated trajectory for the test article, information about the objects to be released from the test article, information about the drop zone, and an atmospheric data file.

1. Simulated Trajectory

Prior to every test, simulations are run to predict the trajectory of the test article, including descent rate, parachute loads, and dynamic pressures. The simulation tools have been tested and used extensively, while the parachute models are continuously being improved based on test data; the results of the simulations are sufficiently accurate to provide a good basis for the footprint prediction. The altitude and velocity time histories output by any of the CPAS simulations can be used in Sasquatch. The data is either loaded into MATLAB using a script or by manually copying and pasting it into arrays. The data is then saved into MATLAB data files to be loaded by Sasquatch.

\section{Object Information}

Before running Sasquatch for a given test, a MATLAB file containing information about the released items must be created. This file contains two objects: a cell array of object names, used for plotting in Sasquatch, and a numerical array of object information. The latter array, called 'objdata', has a row for each object, populated with that object's weight, drag area, release altitude (the altitude at which it is cut away or released from the test article), drag modification factor (used to adjust the object's descent rate to match past test data), and mortar velocities (for mortar lids and sabots). Optionally, a row may be included for the payload itself, to perform a calculation of the payload landing location in the event of a ballistic scenario (i.e. with no parachutes on the payload).

\section{Drop Zone Information}

The user is able to specify a drop zone at the initialization of the program. Information for common drop zones is saved in MATLAB data files. Sasquatch loads the appropriate file based on the user input. These files contain boundary information, a calculated center point of the drop zone, and the mean sea level altitude at ground level.

\section{Atmospheric Data}

Day of test wind and atmosphere information is key to footprint calculations. The wind direction and speed dictates the horizontal motion of the objects, while the air density affects the descent rates. Sasquatch reads in the Rawinsonde data files, which contain the direction and speed of the winds at various altitudes, as well as the air density, temperature, and pressure, usually in increments of $100 \mathrm{ft}$ of altitude.

\section{Modification of Payload Trajectory and Determination of Safe Aircraft Release Point}

After the appropriate files have been read, Sasquatch perturbs the test article trajectory based on the wind data. It is assumed that the payload, as well as all released objects, moves "with the wind" - that is, at any given altitude, the horizontal velocity of the payload is equal to the measured wind velocity at that altitude, in both magnitude and direction.

An initial horizontal velocity is added to the payload's trajectory, to account for the velocity imparted by the carrier aircraft. The "horizontal throw" is the time it takes for the payload to bleed off that imparted velocity. The horizontal throw is calculated based on the drag of the payload, the aircraft velocity at extraction/release, the air density at altitude, and the wind velocity at altitude. First, the acceleration is calculated:

$$
a=\frac{\frac{1}{2} \rho v_{0}^{2} C_{D} S g}{W}
$$

Where $a$ is the horizontal acceleration, $\rho$ is the atmospheric density, $v_{0}$ is the initial horizontal velocity, $C_{D} S$ is the drag area of the payload, $g$ is the acceleration due to gravity, and $W$ is the payload mass.

Then, the velocity with respect to time is calculated, assuming a linear slope:

$$
v(t)=v_{\text {wind }}+v_{0}-a t
$$

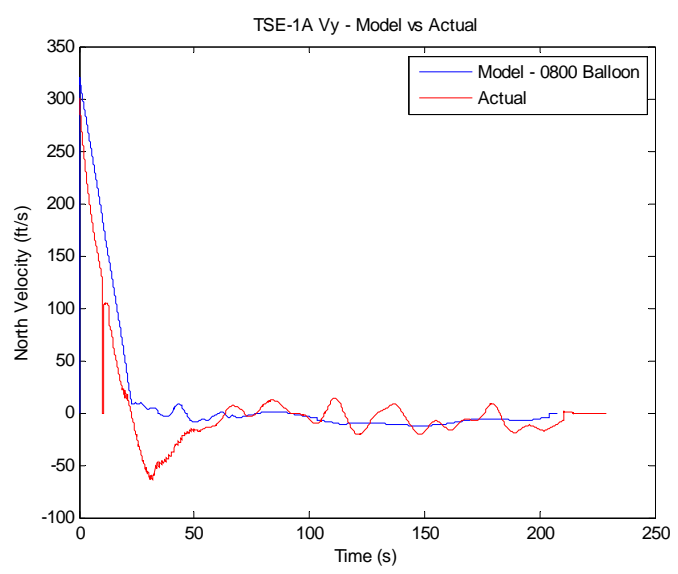

Figure 4. Comparison of Sasquatch horizontal throw calculation with actual flight data. 
The horizontal throw model matches well with the deceleration seen on actual flight data (Fig. 4).

Once a final trajectory is established, Sasquatch sets the landing location of that trajectory in the center of the drop zone or another specified location. The aircraft release point is then calculated based on that landing location. Alternatively, the user can specify an aircraft release point, and Sasquatch will calculate the landing location based on that point. (This option is typically used when wind data is received after a release point has already been established, to ensure that the updated trajectory will still keep the payload within the range.)

\section{Released Item Trajectories and Landing Locations}

After the aircraft release point and test article landing location have been determined, a trajectory is calculated for each of the objects released from the test article during flight. For the most part, these objects are parachutes; on tests using mortars, trajectories for mortar sabots and lids are also determined. The steady state vertical velocity for an object is calculated based on its weight and drag area. Because the drag area of an unloaded canopy is difficult to determine, a drag modification factor is used to adjust the drag area such that the resulting vertical velocity matches data from previous flight tests. That is:

$$
C_{D} S=C_{D} S_{\text {full canopy }} * \text { factor }
$$

And

$$
v=-\sqrt{\frac{2 m}{\rho C_{D} S}}
$$

Where $C_{D} S$ is the object's drag area, ${ }^{5} v$ is the vertical velocity, $m$ is the weight of the object, and $\rho$ is the initial atmospheric density.

Like the payload, all released objects are assumed to move horizontally with the wind. The horizontal velocity at any given time is the same as the wind velocity at the object's current altitude. The change in position is calculated using a simple linear assumption:

$$
x=v_{x} d t+x_{n-1}
$$

Where $x$ is the new position, $v_{x}$ is the horizontal velocity, $d t$ is the program's time step, and $x_{n-1}$ is the current position.

The program iterates until an object reaches the ground. Sasquatch considers each object sequentially, proceeding in order through the rows of the 'objdata' array.

\section{E. Dispersions}

The uncertainties of the inputs and the actual test environment must be accounted for in the predictions. To do this in Sasquatch, dispersion circles are set around the landing location for each object, including the test article. These dispersions are based on uncertainties in release point, winds, and descent rates. The dispersion radius is calculated as follows:

$$
r_{f t}=T * \operatorname{disp}_{R O D} * \operatorname{disp}_{\text {wind }}
$$

Where $r_{f t}$ is the object's dispersion radius in feet, $T$ is the total time aloft for that object, $\operatorname{disp}_{R O D}$ is a dispersion on

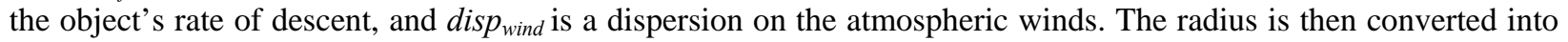
degrees latitude and a release point dispersion is added. Current uncertainties are 10 seconds of latitude and longitude for the release point; $10 \mathrm{ft} / \mathrm{s}$ in all directions for the winds; and $20 \%$ on the descent rate. These dispersions are based on actual variations seen on past tests. They will be reconsidered for future versions of Sasquatch.

\section{F. Outputs}

After landing locations have been calculated for all objects, the results are collected, saved, and output to the screen. The output is in two parts: text and graphical. 
The graphical output of highest importance is the map (Fig. 5). The appropriate drop zone for the test is mapped, along with all relevant boundaries and safety zones. The predicted landing locations are then plotted on that map, with a different color representing each object. Dispersions are shown as circles centered on each object's landing location. The radius of each circle depends on the formulas described above; specifically, the longer an object is predicted to be in the air, the larger its dispersion circle will be. The aircraft run-in and release point are also marked.

The output map is used to assess the feasibility of the current footprint. For an acceptable footprint, these conditions must be true:

- $\quad$ All objects are on the range.

- No dispersion circles are over the eastern edge (public highway).

- The load is on the cleared area.

- The release point and aircraft run-in are not endangering a KTM camera site.

If any of those conditions are not true, an alternate solution must be found. Sasquatch allows the user to shift the release point in latitude and longitude, thereby shifting the entire footprint. In some cases, this is sufficient to bring all objects onto the range while still keeping the payload on the cleared area. In other cases, shifting the release point does not resolve the issue. In those cases, if possible, the release altitude is lowered. Preprepared "wind contingency" trajectories covering the acceptable range of release altitudes are typically prepared before the test. When necessary, Sasquatch can be re-run using one of those wind contingency cases, in hopes of achieving a solution that allows a test on that day.

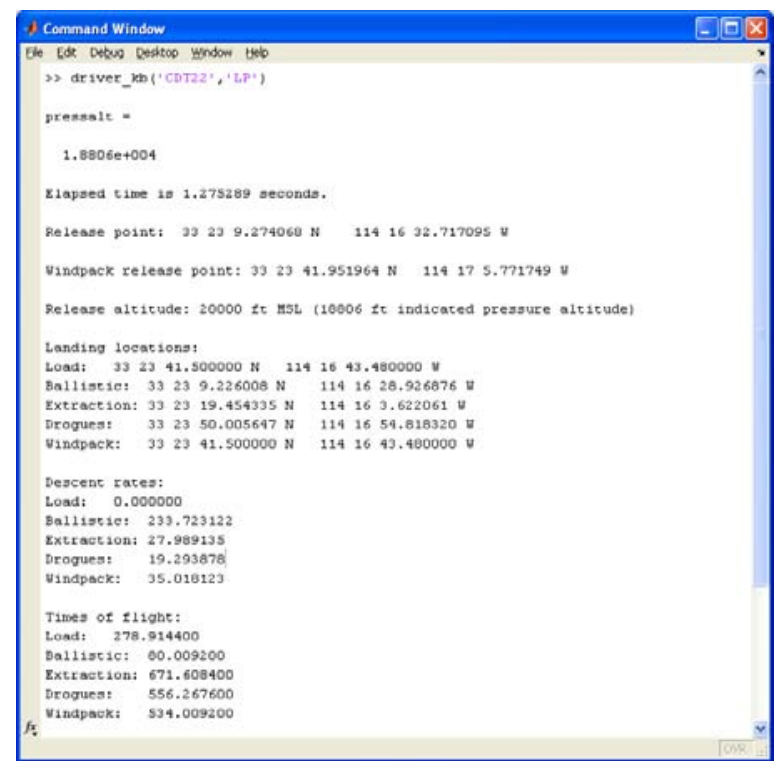

Figure 6. Sample text output.

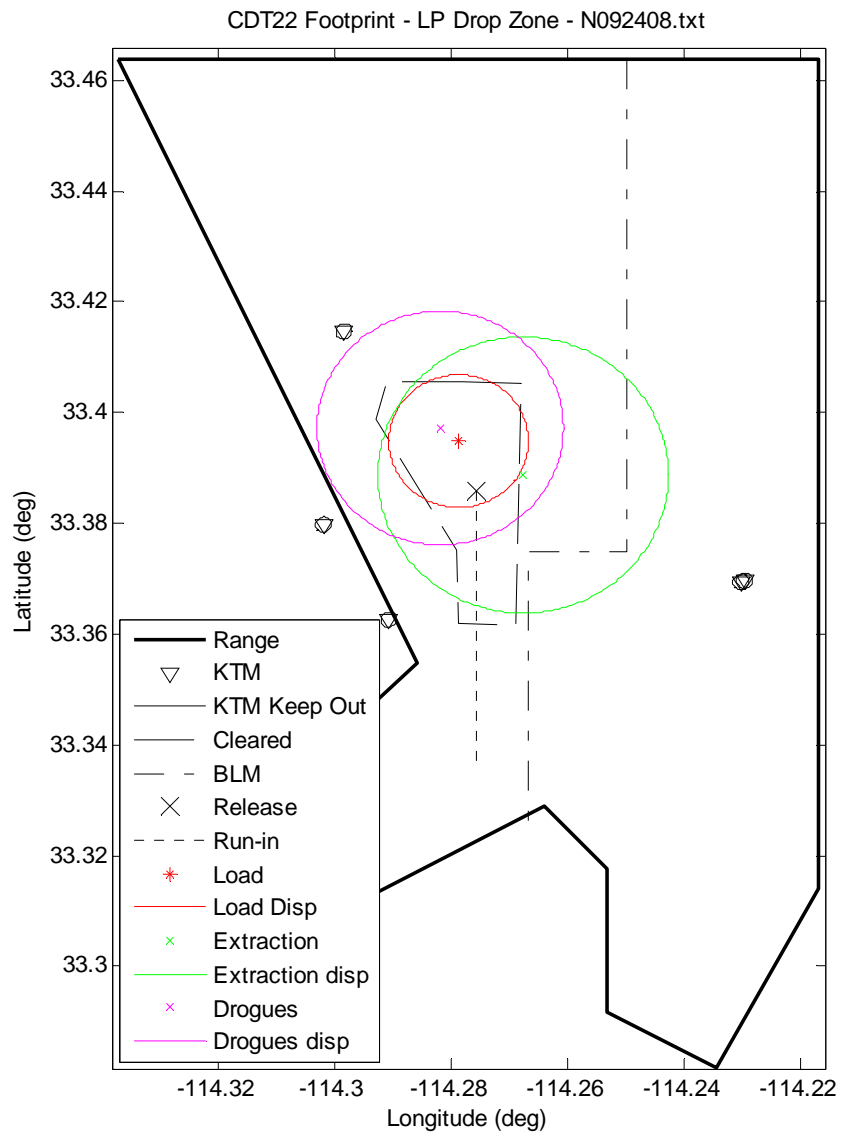

Figure 5. Sample output map.
Once a solution is determined, relevant information is output to the MATLAB command window (Fig. 6): release altitude (both geometric and pressure altitudes), the release point and landing locations in degrees, minutes, and seconds latitude and longitude, and the descent rates and total times of flight for each released object. These outputs can be used as test documentation as well as for communication of the final release point and release altitude.

\section{Validation}

To date, Sasquatch has been used on thirteen CPAS drop tests. After each test, actual landing locations of the payload and all released objects are recorded. Two comparisons are used to qualitatively determine the accuracy of the tool: a comparison of the actual landing locations to the preflight predictions, and a comparison of the actual locations to a prediction updated with postflight atmospheric conditions and the actual aircraft release point. The current version of Sasquatch produces predictions that correlate well with the test data. 
In 2009, improvements were made to the horizontal throw model and the dispersion circles. Data from several tests were used to test the new models. Figures 7 and 8 show the results of preflight and postflight Sasquatch runs compared with the actual landing locations for CDT-3, a Generation I cluster test. The preflight run uses the release point that was given to the aircrew and the final preflight weather balloon data. With those inputs, the prediction is south of the actual landing location, but the latter falls within the dispersion circle (Fig. 7).

The postflight run uses the actual aircraft release point, taken from GPS data, and the postflight weather balloon data, which is closer in time to the actual test than the preflight balloon. The postflight run accounts for most of the uncertainty and yields a result close to the actual landing location (Fig. 8).

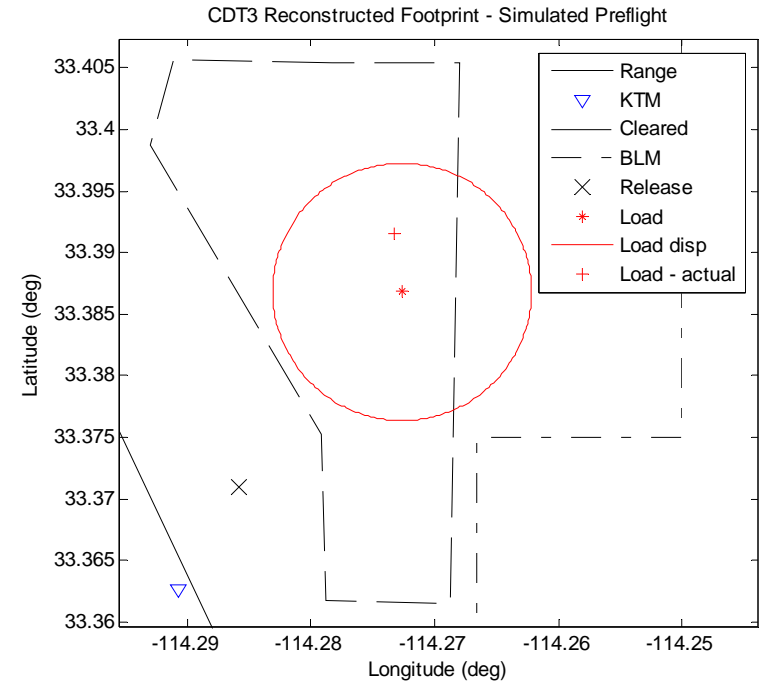

Figure 7. CDT-3 Preflight Prediction

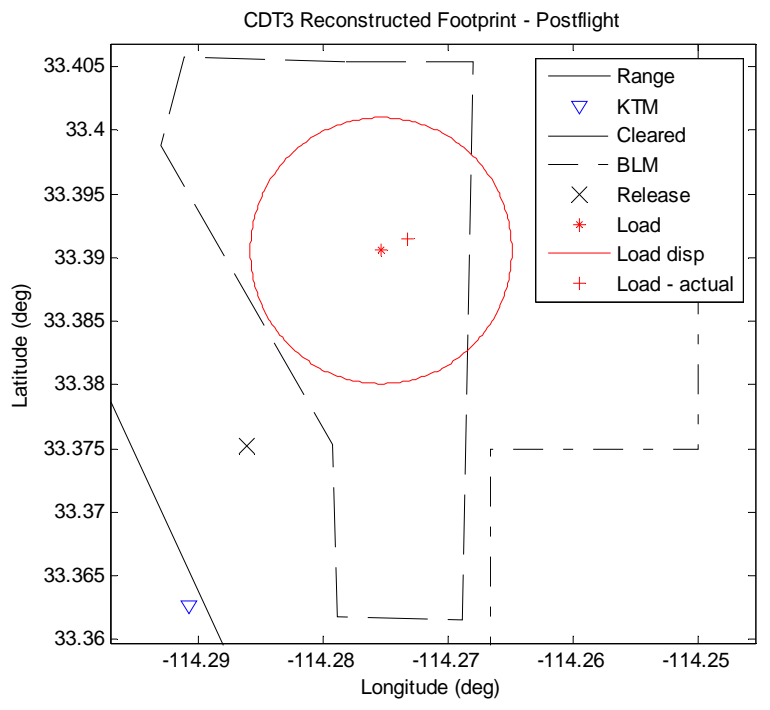

Figure 8. CDT-3 Postflight Comparison

The current version of Sasquatch was used for the day-of-test predictions for all Generation II tests, which were executed throughout 2010. On all six of these tests, using release points determined in Sasquatch, the payloads landed within the targeted cleared area of the drop zone and all released objects stayed on the range. On CDT-2-1 and CDT-2-2, two Generation II cluster tests, the payload landed very close to the predicted landing location (Fig. 9, 10). The released objects landed farther away from the center of their dispersions, but still within the dispersion radius. The larger difference between predictions and actual landing locations is due to the lower understanding of the descent rate of unloaded parachutes as well as the longer time aloft of those objects; shifts in the wind profile have more time to affect their trajectories. The dispersion circles account well for those potential shifts.
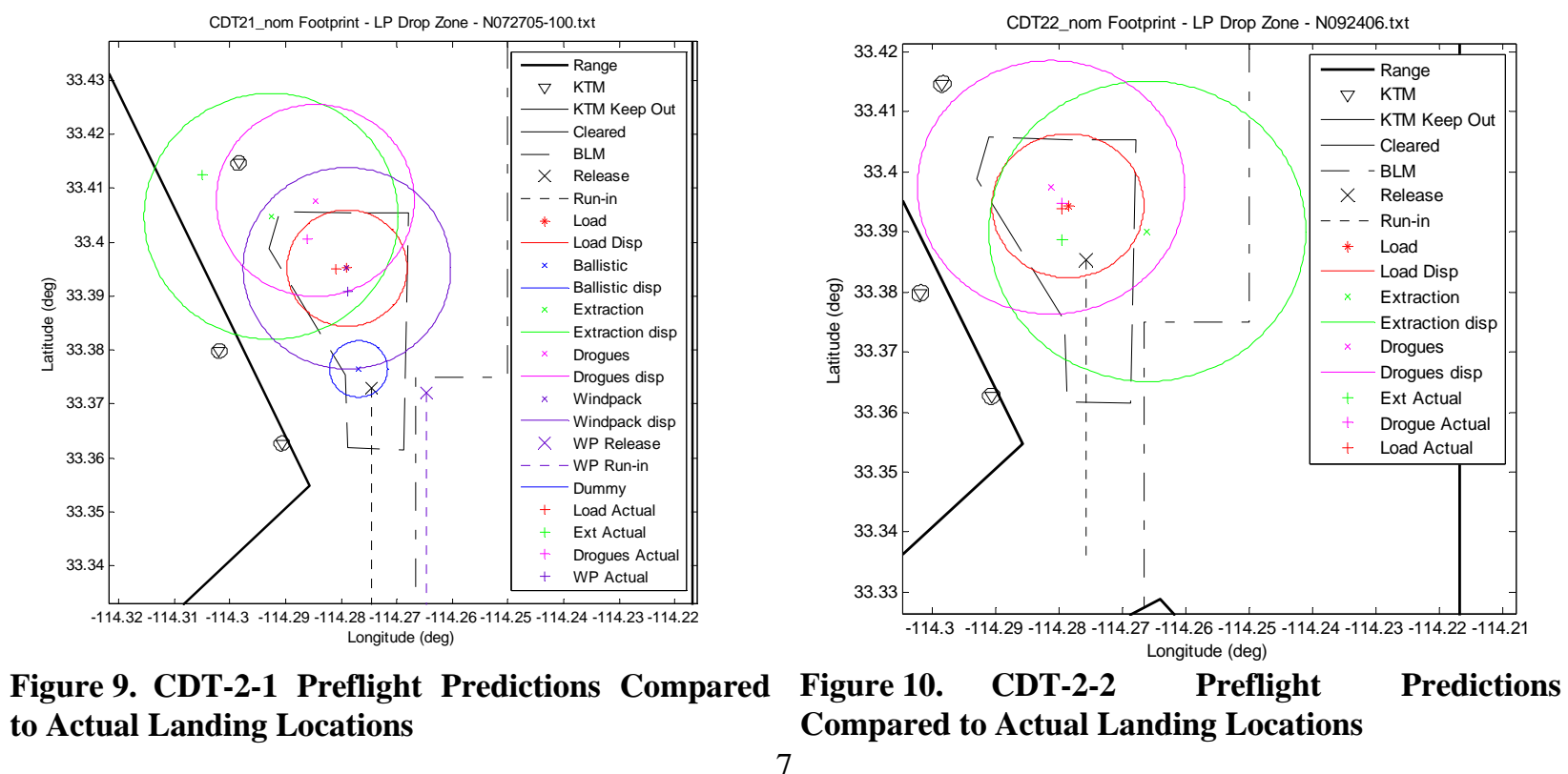

American Institute of Aeronautics and Astronautics 


\section{Future Improvements}

Although the current version of the tool provides well-correlated predictions, some improvements can be made. The current level of uncertainty yields dispersion circles that sometimes strictly limit the altitude at which the test article can be released. The requirement is for all dispersions to stay within the range, with the majority of the payload dispersion circle contained on the cleared area. On a day with even moderate winds, the dispersions circles become unacceptable when releasing the payload from 20,000 ft or higher. Investigation will be made to potentially decrease or modify the dispersions used in Sasquatch. One possibility is that the shape of the possible landing locations should be ovoid or teardrop-shaped, rather than circular. Such a shape would cover less ground area and therefore decrease the likelihood of impinging on the edge of the range. In order to determine an appropriate shape, a study will have to be done wherein the winds and release point are varied and the effects assessed.

Other changes, such as the addition of a graphical user interface and the incorporation of Google Earth imagery, are also planned.

\section{Conclusions}

CPAS has developed the Sasquatch footprint tool to ensure range safety during parachute drop tests. This tool incorporates simulated trajectories, object information, and local atmospheric data to predict the landing locations of test payloads and all released objects. Sasquatch is well-correlated to test data and continues to ensure the safety of test personnel as well as the safe recovery of all equipment. The tool will be modified as needed based on new test data, improving predictions and providing capability for more complex tests as CPAS moves into its next generation of testing.

\section{References}

${ }^{1}$ Lichodziejewski, D., Taylor, T., Sinclair, R., Olmstead, R., Kelley, C., Johnson, J., Melgares, M., Morris, A., and Bledsoe, K., "Development and Test of the Orion Parachute Assembly System,” AIAA-2009-16640, May 2009.

${ }^{2}$ Bledsoe, K., Englert M., Morris, A., and Olmstead, R., "Overview of the Crew Exploration Vehicle Parachute Assembly System (CPAS) Generation I Main and Cluster Development Test Results,” AIAA-2009-64906, May 2009.

${ }^{3}$ Olmstead, R. Morris, A., Bledsoe, K., and Englert, M., "Overview of the Crew Exploration Vehicle Parachute Assembly System (CPAS) Generation I Drogue and Pilot Development Test Results,” AIAA-2009-164897, May 2009.

${ }^{4}$ Rogers, J., “Comparative Analysis Involving Wind Profile Data Sources,” AIAA 2009-2960, May 2009.

${ }^{5}$ Knacke, T.W., Parachute Recovery Systems, Para-Publishing, Santa Barbara, CA, 1992. 